\title{
Erratum: Depth Dose Measurement using a Scintillating Fiber Optic Dosimeter for Proton Therapy Beam of the Passive-Scattering Mode Having Range Modulator Wheel
}

\author{
[J. Korean Phys. Soc. 72, 1025 (2018)] \\ DOI: $10.3938 / \mathrm{jkps} .72 .1025$ \\ Ui-Jung HwANG \\ Department of Radiation Oncology, National Medical Center, Seoul 04564, Korea \\ Dongho Shin, Se Byeong LeE, Young Kyung Lim, Jong Hwi Jeong and Hak Soo Kim \\ Proton Therapy Center, National Cancer Center, Goyang 10408, Korea \\ Ki Hwan KIM* \\ Department of Radiation Oncology, Chungnam National University Hospital, Daejeon 35015, Korea \\ DOI: $10.3938 /$ jkps. 72.1271
}

The affiliation of Ui-Jung Hwang was incorrect. It should be corrected as "Department of Radiation Oncology, Chungnam National University Hospital, Daejeon 35015, Korea".

*E-mail: khkim@cnuh.co.kr; Fax: +82-42-280-7889 